\title{
FUNCTION ALGEBRAS AND FLOWS. IV
}

\author{
BY \\ PAUL S. MUHLY( ${ }^{1}$ )
}

\begin{abstract}
The automorphisms of the algebra $\mathscr{U}$ of analytic functions associated with a flow (without periodic orbits) are completely determined. This result extends earlier work of Arens who determined the automorphisms of when the flow is almost periodic. The Choquet boundary of the maximal ideal space of $\mathfrak{a}$ is also determined under the hypothesis that the flow has no fixed points.
\end{abstract}

1. Introduction. As in the other papers in this series (see [8], [9], [10]), $X$ will be a fixed compact Hausdorff space upon which the real line $\mathbf{R}$ acts continuously as a topological transformation group. The pair $(X, \mathbf{R})$ will be referred to as a flow. If $x$ is in $X$ and if $t$ is in $R$, then $x+t$ will denote the translate of $x$ by $t$. A continuous complex-valued function $\phi$ on $X$ is called analytic in case for each $x$ in $X$ the function of $t, \phi(x+t)$, belongs to $H^{\infty}(\mathrm{R})$, the space of bounded measurable functions on $\mathrm{R}$ which admit bounded analytic extensions into the upper half-plane. The algebra of all analytic functions on $X$ will be denoted by $\mathscr{A}$. Our objective in this article is to determine all the automorphisms of $\mathfrak{\mathscr { A }}$.

When $X$ is the circle $\mathbf{T}$ and $\mathbf{R}$ acts on $X$ in the usual fashion, $\mathscr{A}$ is the disc algebra. In this case it is well known that an automorphism of $\mathscr{A}$ is induced via composition with the restriction to $\mathbf{T}$ of a fractional linear transformation of the unit disc onto itself. If $X$ is an almost periodic compactification of $\mathbf{R}$, then $\mathscr{U}$ may be regarded as the space of analytic almost periodic functions on $\mathbf{R}$ whose Bohr-Fourier coefficients are supported in the nonnegative half of a dense subgroup $\Gamma$ of $\mathbf{R}$. In this case Arens [1] showed that every automorphism of $\mathfrak{A}$ is induced via composition with an affine map of $X$ such that the adjoint of the additive factor of this map preserves the order on $\Gamma$. Our principal result, Theorem III, is a generalization of this. It asserts that if the flow has no periodic orbits, then each automorphism of $\mathscr{Q}$ is induced via composition with a homeomorphism $\sigma$ of $X$ for which there is a positive,

Received by the editors May 3, 1973.

AMS (MOS) subject classifications (1970). Primary 46J 10, 46J 15, 46J 35, 30A98; Secondary 22D40, 54H 20.

(1) This research was supported in part by the National Science Foundation. 
continuous, invariant function $\beta$ on $X$ such that $\sigma(x)+t=\sigma(x+\beta(x) t)$ for all $x$ in $X$ and all $t$ in $\mathrm{R}$.

Observe that if $X$ is the circle, then a homeomorphism which intertwines the action of $\mathbf{R}$ in the fashion just described necessarily is itself a rotation. Thus, in a sense, the disc algebra is exceptional; its group of automorphisms is much richer than that of the algebra of analytic functions associated with a flow without periodic orbits. We intentionally avoid flows with periodic orbits for two reasons. The first is that since the restriction of the functions in $\mathcal{U}$ to a periodic orbit yields a copy of the disc algebra and since the disc algebra is well understood, nothing is lost by ruling out periodic orbits. The second reason is that since periodic orbits, if they exist, may be imbedded in $X$ in rather wild ways, particularly if there is a large number of them, and since this makes the analysis virtually intractable, there is a great deal to be gained by ruling out periodic orbits.

In the next section we present some preliminary material which we shall need. In $\S 3$ we show that if there are no points in $X$ left fixed by the action of $\mathbf{R}$, then the Choquet boundary of the maximal ideal space $M_{\mathfrak{I}}$ of $\mathfrak{I}$ is $X$. This will be used in the proof of Theorem III. Also in $\S 3$, we extend some of our earlier work concerning the structure of $M_{\mathfrak{x}}$. Our characterization of the automorphisms of $\mathcal{A}$, Theorem III, is presented in $\S 4$. $\$ 5$ is devoted to corollaries of Theorem III; one of these is Arens' result. In $\S 6$ we close with a conjecture for future investigation.

2. Preliminaries. When it is convenient, we will often write $T_{t} x$ for $x+t, x \in X, t \in \mathrm{R}$, and regard $\left\{T_{t}\right\}_{t \in \mathrm{R}}$, as a continuous one-parameter group of homeomorphisms of $X$. Also, we will regard $\left\{T_{t}\right\}_{t \in R}$ as acting on functions or on measures on $X$, but we shall not distinguish notationally between the various roles of $\left\{T_{t}\right\}_{t \in \mathrm{R}}$. Thus if $\phi$ is a function, $T_{t} \phi$ will denote the function whose value at $x$ is $\phi\left(T_{t} x\right)$, while if $\mu$ belongs to $M(X)$, the space of all finite regular Baire measures on $X$, then $T_{t} \mu$ will denote the measure whose value at the Baire set $E$ is $\mu\left(T_{-t} E\right)$. We note that our notation is slightly different from that in our other papers on flows where $T_{t} \phi$ is what we would now write as $T_{-t} \phi$.

When restricted to $C(X)$, the space of continuous complex-valued functions on $X,\left\{T_{t}\right\}_{t \in \mathrm{R}}$ constitutes a strongly continuous one-parameter group of automorphisms and so, in the usual fashion, $C(X)$ and $M(X)$ may be converted into modules over the group algebra $L^{1}(R)$ via the following formulae: If $f$ is in $L^{1}(\mathrm{R})$ and if $\phi$ is in $C(X)$, then we write $\phi * f$ for the value of the Bochner integral $\int_{-\infty}^{\infty} T_{-t} \phi f(t) d t$; while if $\mu$ is in $M(X)$, we write $\mu * f$ for the measure such that $\int \phi d(\mu * f)=\int(\phi * \widetilde{f}) d \mu$ for all $\phi$ in $C(X)$ where 
$\widetilde{f}(t)=f(-t)$. If $\rho$ is a function in $C(X)$ or a measure in $M(X)$, then the spectrum of $\rho$, denoted by $\operatorname{sp}(\rho)$, is defined to be the hull of the ideal consisting of those functions $f$ in $L^{1}(\mathbf{R})$ such that $\rho * f=0$. We refer the reader to [4] for the basic facts about spectra.

The algebra $\mathscr{A}$ may be described as the space of functions $\phi$ in $C(X)$ such that $\operatorname{sp}(\phi) \subseteq[0, \infty)$. The following lemma is implicit in some of our earlier work as well as in the work of others. We present it and its proof here for the sake of completeness.

Lemma 2.1. Let $\mu$ be a real measure in $M(X)$. Then $\mu$ annihilates every continuous function with strictly positive spectrum if and only if $\mu$ is invariant.

Proof. If $\mu$ annihilates every such function, then $\operatorname{sp}(\mu) \subseteq[0, \infty)$ by Proposition 2 of [4]. On the other hand, since $\operatorname{sp}(\bar{\phi})=-\operatorname{sp}(\phi)$ for all $\phi$ in $C(X)$ (see $[4$, p. 48]), the fact that $\mu$ is real implies that $\mu$ annihilates every continuous function with strictly negative spectrum as well. Therefore, by Propostion $2^{\prime}$ of [4], $\operatorname{sp}(\mu) \subseteq(-\infty, 0]$. Hence $\operatorname{sp}(\mu) \subseteq\{0\}$ and, by Proposition 2.2 of [8], $\mu$ is invariant. The converse follows immediately from Proposition 2.2 of [8] and Proposition 2 of [4].

A measure $\mu$ in $M(X)$ is called quasi-invariant in case its total variation measure $|\mu|$ and all its translates $T_{t}|\mu|, t \in R$, have the same null sets. We note for later use the fact that if $\mu$ is a positive quasi-invariant measure in $M(X)$, then the restriction of $\left\{T_{t}\right\}_{t \in R}$ to $L^{\infty}(\mu)$ constitutes a weak-* continuous, one-parameter group of *automorphisms of $L^{\infty}(\mu)$. This follows easily from [5, Proposition 1]. We also note that if $\mu$ is quasi-invariant, then the map $t \rightarrow T_{t} \mu$ from $\mathrm{R}$ into $M(X)$ is continuous when $M(X)$ has the total variation measure. This, too, follows from [5, Proposition 1].

Points in the maximal ideal space $M_{\mathfrak{x}}$ of $\mathscr{A}$ will be denoted thus $p$, and representing measures for $p$ will be denoted by $m_{p}$. We shall write $L^{p}\left(m_{p}\right)$ and $H^{p}\left(m_{p}\right)$ for the Lebesgue and Hardy spaces associated with $m_{p}$. The classical spaces on the line $R$ and on the circle $T$ will be denoted by $L^{p}(R)$, $H^{p}(\mathrm{R}), L^{p}(\mathrm{~T})$ and $H^{p}(\mathrm{~T})$.

3. Remarks on the structure of $M_{\mathfrak{x}}$. In [8] we noted that the algebra $\mathfrak{A}$ need not separate the points of $X$ so that, in the strict sense of the term, $\mathscr{A}$ need not be a function algebra on $X$. However, as will be shown presently, under a very mild hypothesis $\mathscr{A}$ does separate the points of $X$.

THEOREM I. If there are no points in $X$ left fixed by the action of $\mathbf{R}$, then $\mathscr{U}$ separates the points of $X$ and $X$, identified in the usual way as a subset of the maximal ideal space $M_{\mathfrak{U}}$ of $\mathfrak{A}$, is the Choquet boundary of $M_{\mathfrak{A}}$. 
Proof. Suppose $\mathscr{A}$ identifies the two points $x$ and $y$ in $X$. Then the real measure $\delta_{x}-\delta_{y}$ annihilates $\mathfrak{A}$ and so by Lemma 2.1 is invariant. (Here, and throughout, $\delta_{x}$ denotes the point mass at $x_{\text {. }}$ ) From this it is readily seen that both $x$ and $y$ are fixed by $\left\{T_{t}\right\}_{t \in R}$ contrary to hypothesis. Thus $\mathscr{A}$ separates the points of $X$.

Since $\mathfrak{A}$ separates the points of $X$, the Choquet boundary of $M_{\mathfrak{X}}$ consists of those points $x$ in $X$ such that the only representing measure for $x$ is $\delta_{x}$. So fix $x$ in $X$ and let $\mu$ be a representing measure for $x$ which is different from $\delta_{x}$. Since $\mathscr{U}$ separates the points of $X, \mu$ is not a point mass $\delta_{y}$ with $y \neq x$. Hence by Theorem III of [8], $\mu$ is quasi-invariant. But also, $\delta_{x}-\mu$ annihilates $\mathcal{U}$ and by Lemma 2.1 must be invariant. Since $\mu$ is quasiinvariant, $T_{t} \mu$ moves continuously in $M(X)$ as $t$ varies over $\mathbf{R}$. Hence so does $\delta_{x+t}=T_{t} \delta_{x}=T_{t}\left(\delta_{x}-\mu\right)+T_{t} \mu$. But, for two distinct points $x$ and $y$ in $X,\left\|\delta_{x}-\delta_{y}\right\|_{M(X)}=2$, so that $T_{t} \delta_{x}=\delta_{x}$ for all $t$. Since this is contrary to hypothesis, we must conclude that the only measure representing $x$ is $\delta_{x}$ and, therefore, that $X$ is the Choquet boundary of $M_{\mathfrak{x}}$. This completes the proof.

Henceforth we will not only assume that there are no fixed points in $X$ but also that there are no periodic orbits in $X$. This means that no $T_{t}$, for $t \neq 0$, has a fixed point. The technical way to say that there are no periodic orbits is to say that $\mathbf{R}$ acts freely on $X$.

In the proof of Theorem I in [9] we showed that if the flow $(X, \mathbf{R})$ is strictly ergodic (meaning that there is only one invariant probability measure on $X$ ) and if $X$ is separable, then certain representing measures for points in $M_{\mathfrak{U}}$ are concentrated on orbits. In the following definition we specify conditions on points $p$ in $M_{\mathfrak{U}}$, where the flow is not necessarily strictly ergodic, which are equivalent to the statement that $p$ has a representing measure concentrated on an orbit (at least if $X$ is separable).

DEFinition 3.1. The set of points $p$ in $M_{\mathfrak{U}}$ satisfying the following three conditions will be denoted by $\mathfrak{T}$ ( $\mathfrak{T}$ is for "thick"):

(a) $p$ has a unique representing measure $m_{p}$,

(b) the Gleason part containing $p$ contains a point different from $p$, and

(c) $m_{p}$ is ergodic.

Observe that conditions (a) and (b) in Definition 3.1 together imply that $m_{p}$ is not a point mass so that, by Theorem III in [8], $m_{p}$ is quasi-invariant. Hence condition (c) in the definition makes sense. The complement of $X \cup \mathcal{I}$ in $M_{\mathscr{Y}}$ is the analogue for flows of what Arens called $\Omega[1]$. When $(X, \mathbf{R})$ is strictly ergodic this complement is the "origin" in the maximal ideal space of थ (see [9]).

We believe that the assumption that $X$ is separable in the second half of 
the next theorem is unnecessary, but at this time we are unable to remove it.

THEOREM II. If point $p$ in $M_{Y} \backslash X$ has a representing measure which is concentrated on an orbit, then $P$ is in $\mathfrak{I}$. If in addition, $X$ is separable, and if $p$ lies in $\mathfrak{I}$, then its unique representing measure is concentrated on an orbit.

Proof. Let $m_{p}$ be a representing measure for $p$ which is concentrated on some orbit 0 . Since $p$ is not in $X, m_{p}$ is not a point mass and so, by Theorem III in [8], $m_{p}$ is quasi-invariant. Moreover, since it is concentrated on an orbit, $m_{p}$ is ergodic. Thus $p$ satisfies condition (c) of Definition 3.1. Suppose $\mu$ is also a representing measure for $p$ and write the Lebesgue decomposition of $\mu$ with respect to $m_{p}$ as $\mu=h m_{p}+\mu_{s}$. Then since $\mu-m_{p}$ annihilates $\mathscr{A}, \mu-m_{p}$ is invariant by Lemma 2.1 , and so its restriction to 0 , which is the restriction of $(h-1) m_{p}$ to 0 , is also invariant. However, 0 is not periodic by our standing hypothesis and, therefore, it does not support any finite invariant measures. Hence $h=1$ a.e. $\left(m_{p}\right)$, and since $m_{p}$ and $\mu$ are probability measures, $\mu_{s}=0$ also. Hence $\mu=m_{p}$ and condition (a) of Definition 3.1 is verified. Finally, for condition (b), it suffices to recall that since $m_{p}$ is supported on an orbit, Theorem I of [9] implies that $m_{p}$ may be written as $\delta_{x} * P_{z}$ for some $x$ in $X$ and some $z$ in the upper half-plane, where $P_{z}(t)=$ $\operatorname{Im}(z) / \pi|z-t|^{2}$ is the Poisson kernel for the upper half-plane. But then, for any other point $w$ in the upper half-plane, $\delta_{x} * P_{w}$ represents a point in $M_{\mathfrak{x}}[9$, Proposition 3.2] which is different from $p$, since $x$ is not fixed [9, Proposition 3.1(c)], and since $\delta_{x} * P_{w}$ is clearly mutually absolutely continuous with respect to $\delta_{x} * P_{z}$, we may conclude that the point represented by $\delta_{x} * P_{w}$ belongs to the Gleason part determined by $p$. Thus condition (b) is verified, and the proof of the first half is complete.

For the qualified converse, let $p$ lie in $\mathfrak{I}$ and let $m_{p}$ be its unique representing measure. Conditions (a) and (c) in Definition 3.1 coupled with Corollary 3.1 of [8] imply that $H^{\infty}\left(m_{p}\right)$ is a maximal weak-* closed subalgebra of $L^{\infty}\left(m_{p}\right)$. Since the Gleason part containing $p$ is larger than $\{p\}$ we may apply a theorem of Merrill [7] which is a sharpening of Wermer's imbedding theorem to conclude that there is a Hilbert space isomorphism $W$ from $L^{2}\left(m_{p}\right)$ onto $L^{2}(\mathrm{~T})$ which carries $H^{2}\left(m_{p}\right)$ onto $H^{2}(\mathrm{~T})$ such that $W L^{\infty}\left(m_{p}\right) W^{-1}=$ $L^{\infty}(\mathrm{T})$ and $W H^{\infty}\left(m_{p}\right) W^{-1}=H^{\infty}(\mathrm{T})$. At this point the remainder of the proof is the same as Step 1 in the proof of Theorem I in [9] and so will be omitted.

4. Automorphisms. In this section we present our theorem describing the automorphisms of $\mathscr{H}$. Our basic assumption that the action of $\mathbf{R}$ on $X$ is free is still in force so that, by Theorem I, $X$ is the Choquet boundary of $M_{\mathfrak{x}}$. We will use the same letter to denote a function in $\mathfrak{A}$ and its Gelfand transform on $M_{\mathfrak{n}}$. 
If $U$ is an automorphism of $\mathscr{A}$, then according to a theorem of Nagasawa [11], there is a homeomorphism $\tilde{\sigma}$ of $M_{\mathfrak{x}}$ onto $M_{\mathfrak{x}}$ such that for all $\phi$ in $\mathscr{U}$ and all $p$ in $M_{\mathfrak{x}},(U \phi)(p)=\phi(\tilde{\sigma}(p))$. The homeomorphism $\tilde{\sigma}$ is simply the restriction of the adjoint $U^{*}$ of $U$ to $M_{\mathfrak{n}}$ where $M_{\mathfrak{I}}$ is regarded as a subset of the dual $\mathfrak{A}^{*}$ of $\mathfrak{A}$. Since the Choquet boundary of $M_{\mathfrak{U}}$, which is $X$, may be identified as the set of extreme points in $\left\{L \in \mathscr{Q}^{*} \mid\|L\|=L(1)=1\right\} \quad[6, \mathrm{p}$. 49], it follows that $\tilde{\sigma}$ carries $X$ onto $X$. We shall denote the restriction of $\tilde{\sigma}$ to $X$ by $\sigma$. Via $\sigma$, we may extend $U$ to all of $C(X)$ by setting $(U \phi)(x)$ $=\phi(\sigma x), \phi \in C(X)$. The following lemma, whose proof is easy and so will be omitted, allows us to extend $U$ to other spaces as well.

LEMMA 4.1. If $m_{p}$ is a representing measure for a point $p$ in $M_{\mathfrak{x}}$, then $m_{p} \circ \sigma^{-1}$ represents $\tilde{\sigma}(p)$.

It follows from the lemma that for each $p$ in $M_{\mathfrak{I}}$ and each representing measure $m_{p}$ for $p, U$ may be extended to an isometric isomorphism from $L^{p}\left(m_{p}\right)$ onto $L^{p}\left(m_{p} \circ \sigma^{-1}\right)$ for all $p, 1 \leqslant p \leqslant \infty$. The extension is given by the formula $(U f)(x)=f(\sigma(x)), f \in L^{p}\left(m_{p}\right)$. We will keep the same letter for all such possible extensions because in context no confusion should arise. Observe that $U H^{p}\left(m_{p}\right)=H^{p}\left(m_{p} \circ \sigma^{-1}\right), 1 \leqslant p \leqslant \infty$, also.

LEMmA 4.2. Suppose the representing measure $m_{p}$ for a point $p$ in $\mathfrak{T}$ is supported on the orbit through the point $x$ in $X$. For $\phi$ in $L^{\infty}\left(m_{p}\right)$ set $(V \phi)(s)=\phi(x+s), s \in \mathbf{R}$ Then $V$ is a weak-* continuous *-isomorphism of $L^{\infty}\left(m_{p}\right)$ onto $L^{\infty}(\mathbf{R})$ which carries $H^{\infty}\left(m_{p}\right)$ onto $H^{\infty}(\mathbf{R})$.

Proof. We noted earlier that $m_{p}$ can be written as $\delta_{x} * P_{z}$ for some $z$ in the upper half-plane. Since $P_{z}(t)>0$ for all $t$, it follows that $V$ is a weak-* continuous *-isomorphism of $L^{\infty}\left(m_{p}\right)$ onto $L^{\infty}(\mathbf{R})$. Since each function in $H^{\infty}\left(m_{p}\right)$ is the almost everywhere limit of a sequence in $\mathcal{U}$, it follows that $V$ carries $H^{\infty}\left(m_{p}\right)$ into $H^{\infty}(\mathrm{R})$. Since $H^{\infty}\left(m_{p}\right)$ is a maximal weak-* closed subalgebra of $L^{\infty}\left(m_{p}\right)$ by Theorem II and Corollary 3.1 of [8] and since $H^{\infty}(\mathbf{R})$ is weak-* closed in $L^{\infty}(\mathbf{R})$, it follows that $V$ carries $H^{\infty}\left(m_{p}\right)$ onto all of $H^{\infty}(\mathrm{R})$.

We turn now to the main theorem of this paper.

THEOREM III. Suppose the action of $\mathbf{R}$ on $X$ is free and let $U$ be an automorphism of 2 . Then there is a homeomorphism $\sigma$ from $X$ onto $X$ and a positive, continuous, invariant function $\beta$ on $X$ such that

$$
\sigma^{-1} T_{t} \sigma(x)=T_{\beta(x) t}(x)
$$

for all $t$ in $\mathrm{R}$ and $x$ in $X$, and such that 


$$
(U \phi)(x)=\phi(\sigma(x))
$$

for all $\phi$ in $\mathcal{A}$ and $x$ in $X$. Conversely, given a homeomorphism $\sigma$ from $X$ onto $X$ and a positive, continuous, invariant function $\beta$ on $X$ such that equation (4.1) holds, then $U$, defined by equation (4.2), is an automorphism of $\mathscr{Y}$.

Proof. We have already indicated that if $U$ is an automorphism of $\mathscr{A}$, then $U$ is implemented via equation (4.2) by a homeomorphism $\sigma$ from $X$ onto $X$. Hence it suffices to produce a positive, continuous, invariant function $\beta$ on $X$ such that equation (4.1) holds. Fix $x$ in $X$ and let $p$ be a point in $\mathcal{I}$ whose representing measure $m_{p}$ is supported on the orbit through $x$. (The point represented by $\delta_{x} * P_{i y}, y>0$, will do.) Regard $\left\{T_{t}\right\}_{t \in \mathrm{R}}$ as acting on functions and recall that for each $q$ in $M_{\mathfrak{U}} \backslash X$, each representing measure $m_{q}$ for $q$ is quasi-invariant [8, Theorem III]. Hence when restricted to $L^{\infty}\left(m_{q}\right)$, $\left\{T_{t}\right\}_{t \in \mathrm{R}}$ constitutes a weak-* continuous one-parameter group of *automorphisms of $L^{\infty}\left(m_{q}\right)$ which fixes $H^{\infty}\left(m_{q}\right)$. From this and the opening remarks of this section, it follows that when $U$ is regarded as a map from $L^{\infty}\left(m_{p}\right)$ onto $L^{\infty}\left(m_{p} \circ \sigma^{-1}\right)$, the family $\left\{U T_{t} U^{-1}\right\}_{t \in \mathrm{R}}$ also constitutes a weak-* continuous one-parameter group of *automorphisms of $L^{\infty}\left(m_{p}\right)$ which fixes $H^{\infty}\left(m_{p}\right)$. Consequently, if $V$ is the isomorphism from $L^{\infty}\left(m_{p}\right)$ onto $L^{\infty}(\mathbf{R})$ given in Lemria 4.2, then $\left\{V U T_{t} U^{-1} V^{-1}\right\}_{t \in \mathrm{R}}$ is a weak-* continuous one-parameter group of *automorphisms of $L^{\infty}(\mathrm{R})$ which fixes $H^{\infty}(\mathrm{R})$. Therefore, according to a theorem of deLeeuw, Rudin, and Wermer [2], there is a one-parameter group $\left\{\alpha_{t}\right\}_{t \in R}$ of fractional linear transformations of the upper half-plane onto itself such that $V U T_{t} U^{-1} V^{-1} \phi=\phi \circ \alpha_{t}$ for all $\phi$ in $L^{\infty}(\mathrm{R})$ and all $t$ in $\mathbf{R}$. Since, in addition, $\left\{V U T_{t} U^{-1} V^{-1}\right\}_{t \in R}$ is weak-* continuous, $\left\{\alpha_{t}\right\}_{t \in \mathbf{R}}$ is continuous in $t$ as well. Back on $X$, we may conclude that

$$
U T_{t} U^{-1} \phi(x+s)=\phi\left(x+\alpha_{t}(s)\right) \text { a.e. }\left(m_{\rho}\right)
$$

for each $t$ in $\mathbf{R}$ and all $\phi$ in $L^{\infty}\left(m_{p}\right)$. We would now like to assert that when we regard $\left\{T_{t}\right\}_{t \in \mathrm{R}}$ as acting on $X$, then

$$
\sigma^{-1} T_{t} \sigma(x+s)=x+\alpha_{t}(s)
$$

for all $s$ and $t$ in $\mathbf{R}$. For this, fix $t$ in $\mathbf{R}$ and let $s_{t}$ be the point sent to the point at infinity by $\alpha_{t}$. It will develop that this $s_{t}$ is the point at infinity itself, but for the moment pretend that it might not be and let $N_{t}$ be the complement of $\left\{s_{t}\right\}$ in $\mathbf{R}$. Then both sides of equation (4.4) are continuous functions of $s$ for $s$ in $N_{t}$. Since equation (4.3) is valid in particular for continuous functions, it follows from the regularity of $m_{p}$ that $\phi\left(\sigma^{-1} T_{t} \sigma(x+s)\right)=$ $\phi\left(x+\alpha_{t}(s)\right)$ for all continuous functions $\phi$ and all $s$ in $N_{t}$. Since the continuous functions on $X$ separate the points of $X$, we find that equation (4.4) is 
valid for each $s$ in $N_{t}$. Now there are some cases to consider. Observe that since $\left\{\alpha_{t}\right\}_{t \in R}$ is a commuting family of fractional linear transformations, the $\alpha_{t}$ have a set of common fixed points and, moreover, this set coincides with the set of fixed points of any particular $\alpha_{t}$ which is not the identity transformation. Suppose that $\left\{\alpha_{t}\right\}_{t \in R}$ fixes some point $z$ in the finite, closed, upper half-plane. If $\operatorname{Im}(z)>0$, then it is not difficult to see that $\left\{\alpha_{t}\right\}_{t \in R}$ must be periodic so that, for some $t \neq 0, \alpha_{t}$ is the identity transformation. But then, for this $t$, $N_{t}=\mathbf{R}$ and $\sigma^{-1} T_{t} \sigma(x+s)=x+s$ for all $s$ in $\mathbf{R}$. Since this contradicts the freeness of the action of $\mathbf{R}$ on $X$, we must conclude that $\operatorname{Im}(z)=0$. In this case, $z$ belongs to $N_{t}$ for all $t$ so that $T_{t}\left(\sigma^{-1}(x+z)\right)=\sigma^{-1}(x+z)$ for all $t$. Since this, too, contradicts our hypothesis, we find that the only point left fixed by $\left\{\alpha_{t}\right\}_{t \in R}$ is the point at infinity. Hence $N_{t}$ is $\mathrm{R}$ for each $t$, and equation (4.4) is valid for all $t$ and $s$ in $R$. Since the point at infinity is fixed by each $\alpha_{t}$ and since $\left\{\alpha_{t}\right\}_{t \in R}$ is continuous in $t$, there are real numbers $\beta$ and $\gamma$ such that $\alpha_{t}(z)=e^{t \gamma} z+\beta\left(\left(e^{t \gamma}-1\right) / \gamma\right)$ for all $t$ where $\left(e^{t \gamma}-1\right) / \gamma$ is to be interpreted as $t$ if $\gamma=0$ (see [9]). However, if $\gamma \neq 0$, then $\left\{\alpha_{t}\right\}_{t \in R}$ fixes the point $-\beta / \gamma$ in $\mathbf{R}$ which is contrary to what we just showed. Thus, for each $x$ in $X$, we have produced a number $\beta(x)$ in $\mathbf{R}$ such that $\sigma^{-1} T_{t} \sigma(x)=x+\beta(x) t$. Observe that $\beta(x)$ is never zero since there are no fixed points for the action of $\mathbf{R}$ on $X$. Clearly $\beta$, as a function, is invariant. Also, since

$$
\begin{aligned}
\left(U^{-1} \phi\right)(x+t) & =\left(T_{t} U^{-1} \phi\right)(x)=\left[U^{-1}\left(U T_{t} U^{-1}\right) \phi\right](x) \\
& =\phi\left(\sigma^{-1}(x)+\beta\left(\sigma^{-1}(x)\right) t\right)
\end{aligned}
$$

for all $\phi$ in $C(X)$ and since the function of $t,\left(U^{-1} \phi\right)(x+t)$, belongs to $H^{\infty}(\mathrm{R})$ for all $\phi$ in $\mathscr{U}$ because $U^{-1} \phi$ is in $\mathscr{U}$ if $\phi$ lies in $\mathscr{A}$, we find that $\beta$ is positive. For the continuity of $\beta$, let $\left\{x_{\lambda}\right\}$ be a net in $X$ converging to a point $x$ in $X$. To show that $\lim \beta\left(x_{\lambda}\right)=\beta(x)$, it suffices to show that $\left\{\beta\left(x_{\lambda}\right)\right\}$ is bounded. For if it is, then by passing to a subnet if necessary, we may assume that $\lim \beta\left(x_{\lambda}\right)$ exists and is some number $\gamma$ in $\mathbf{R}$. Then, setting $t=1$, we find that

$$
\begin{aligned}
x+\beta(x) & =\sigma^{-1} T_{1} \sigma(x)=\lim \sigma^{-1} T_{1} \sigma\left(x_{\lambda}\right) \\
& =\lim x_{\lambda}+\beta\left(x_{\lambda}\right)=x+\gamma
\end{aligned}
$$

so that, since $\mathbf{R}$ acts freely, $\gamma=\beta(x)$. It follows that if $\left\{\beta\left(x_{\lambda}\right)\right\}$ is bounded, then $\lim \beta\left(x_{\lambda}\right)$ exists and is $\beta(x)$. But if $\left\{\beta\left(x_{\lambda}\right)\right\}$ is unbounded, then, by passing to a subnet if necessary, we may assume that $\lim \beta\left(x_{\lambda}\right)=\infty$. So, since $\sigma T_{1} \sigma^{-1}(y)=y+1 / \beta(y)$ for all $y$ in $X$, we reach the untenable conclusion that 


$$
\begin{aligned}
x & =\lim x_{\lambda}+1 / \beta\left(x_{\lambda}\right)=\lim \sigma T_{1} \sigma^{-1}\left(x_{\lambda}\right) \\
& =\sigma T_{1} \sigma^{-1}(x)=x+1 / \beta(x) .
\end{aligned}
$$

Whence $\beta$ is continuous and the direct half of the proof is complete.

For the converse, suppose that $\sigma$ is a homeomorphism from $X$ onto $X$ for which there is a positive, continuous, invariant function $\beta$ on $X$ such that equation (4.1) holds and let $U$ be defined for all functions $\phi$ in $C(X)$ by equation (4.2). If $\phi$ is in $C(X)$, then for $t$ in $\mathbf{R}$ and $x$ in $X$,

$$
\begin{aligned}
(U \phi)(x+t) & =\left(T_{t} U \phi\right)(x)=U\left(U^{-1} T_{t} U \phi\right)(x) \\
& =\phi(\sigma(x)+t / \beta(\sigma(x))) .
\end{aligned}
$$

Since $\beta$ is positive, $\phi(\sigma(x)+t / \beta(\sigma(x)))$, as a function of $t$, lies in $H^{\infty}(\mathrm{R})$ if $\phi$ is in $\mathscr{A}$. Thus $U$ carries $\mathscr{X}$ into $\mathscr{A}$ and since $U^{-1}$ is easily seen to have the same property, we may conclude that, when restricted to $\mathfrak{A}, U$ is an automorphism. This completes the proof.

5. Corollaries. With a simple change of notation, the proof of Theorem III shows that while the algebra of analytic functions associated with a flow in which $\mathbf{R}$ acts freely does not constitute a complete set of conjugacy invariants for the flow, it does determine the flow up to a trivial change of velocity. We state this formally as

Corollary 5.1. For $i=1,2$, let $\left(X_{i}, \mathbf{R}\right)$ be a flow in which $\mathbf{R}$ acts freely. Denote the group of homeomorphisms on $X_{i}$ determined by $\mathbf{R}$ by $\left\{T_{t}^{(i)}\right\}_{t \in \mathrm{R}}$ and let $\mathfrak{A}_{i}$ be the algebra of analytic functions associated with $\left(X_{i}, \mathbf{R}\right)$. Then the $\mathfrak{A}_{i}$ are isomorphic as algebras if and only if there is a homeomorphism $\sigma$ from $X_{1}$ onto $X_{2}$ and a positive, continuous, invariant function $\beta$ on $X_{1}$ such that $\sigma^{-1} T_{t}^{(2)} \sigma(x)=T_{\beta(x) t}^{(1)}(x)$ for all $t$ in $\mathrm{R}$ and $x$ in $X_{1}$.

The first assertion in the next corollary can be proved directly using Lemma 4.1 without the aid of Theorem III, but with Theorem III it is immediate. Note that it is an analogue for flows of Theorem 4.4 in [1].

COROLlary 5.2. Let $\tilde{\sigma}$ be a homeomorphism of $M_{\mathfrak{x}}$ induced by an automorphism of $\mathcal{U}$ and let $p$ be a point in $M_{\mathfrak{x}}$. Then

(i) $p$ lies in $\mathfrak{T}$ if and only if $\tilde{\sigma}(p)$ lies in $\mathfrak{T}$,

(ii) $p$ has an invariant representing measure if and only if $\widetilde{\sigma}(p)$ has one, and

(iii) $p$ has an ergodic representing measure if and only if $\tilde{\sigma}(p)$ has one.

Since the proof of the corollary is straightforward, it will be omitted. 
In our final corollary we present a new proof of the result of Arens [1] which describes the automorphisms of $\mathscr{Q}$ when the flow $(X, \mathbf{R})$ is minimal and almost periodic but not periodic. In this case, as is well known (see [3]), there is a dense subgroup $\Gamma$ of $R$ such that $X$ is the dual of $\Gamma$ with the discrete topology. The action of $\mathbf{R}$ on $X$ is given by translation with respect to the one-parameter subgroup $\left\{e_{t}\right\}_{t \in R}$ of $X$ whose elements are defined by the equation $\left\langle e_{t}, \gamma\right\rangle=e^{i t \gamma}, \gamma \in \Gamma, t \in \mathrm{R}$, where $\langle$,$\rangle denotes the pairing between$ $X$ and $\Gamma$. The algebra $\mathcal{A}$ of analytic functions on $X$ may then be regarded as the collection of functions $\phi$ in $C(X)$ whose Fourier transforms $\hat{\phi}$ vanish for the negative frequencies in $\Gamma$. Arens' result asserts that every automorphism of $\mathcal{A}$ is given by composition with an affine map of $X$ which preserves the order on $\Gamma$. Here is what this means. An affine map of $X$ is a homeomorphism $\sigma$ of $X$ which may be factored as $\sigma=\sigma_{1} \sigma_{2}$ where $\sigma_{2}$ is an automorphism of $X$ and where $\sigma_{1}$ is translation with respect to some fixed element $y$ of $X$; i.e., $\sigma_{1}(x)=x+y$ for all $x$ in $X$. To say that $\sigma$ preserves the order on $\Gamma$ is simply to say that $\sigma_{2}^{*}$, the adjoint of $\sigma_{2}$ acting on $\Gamma$, maps the semigroup of nonnegative elements in $\Gamma$ onto itself.

Corollary 5.3 (ARENS). Let $\Gamma$ be a dense subgroup of $\mathbf{R}$, let $X$ be the dual of $\Gamma$, let $\mathbf{R}$ act on $X$ in the fashion described above, and let $\mathcal{A}$ be the algebra of analytic functions associated with the flow so obtained. If $U$ is an automorphism of $\mathfrak{Q}$, then there is an order preserving affine map $\sigma$ from $X$ onto $X$ such that

$$
U \phi=\phi \circ \sigma
$$

for all $\phi$ in $\mathfrak{A}$. Conversely, if $\sigma$ is an order presenving affine map of $X$, then $U$, defined by (5.1), is an automorphism of $\mathfrak{A}$.

Proof. Let $U$ be an automorphism of $\mathscr{U}$ and let $\sigma$ be the homeomorphism of $X$ determined by $U$ for which there is a positive, continuous, invariant function $\beta$ on $X$ such that $\sigma^{-1} T_{t} \sigma(x)=T_{\beta(x) t}$ for all $t$ in $\mathbf{R}$. Recall that here $T_{t} x=x+e_{t}$ for all $x$ in $X$ where $\left\{e_{t}\right\}_{t \in R}$ is the oneparameter group described above. Since the flow is minimal (because $\left\{e_{t}\right\}_{t \in R}$ is a dense one-parameter subgroup of $X$ ) the function $\beta$ is a positive constant, say $m$. Let $y=\sigma(0)$, define $\sigma_{1}$ by the formula $\sigma_{1}(x)=x+y$, and set $\sigma_{2}=\sigma_{1}^{-1} \sigma$. Then since $\sigma_{1}$ commutes with $\left\{T_{t}\right\}_{t \in R}$, we find that

$$
\sigma_{2}^{-1} T_{t} \sigma_{2}=\sigma^{-1} \sigma_{1} T_{t} \sigma_{1}^{-1} \sigma=\sigma^{-1} T_{t} \sigma=T_{m t}
$$


for all $t$ in R. Also, since $\sigma_{2}(0)=0$, it follows that $\sigma_{2}^{-1}\left(e_{t}\right)=\sigma_{2}^{-1} T_{t}(0)=$ $\sigma_{2}^{-1} T_{t} \sigma_{2}(0)=T_{m t}(0)=e_{m t}$. Thus when restricted to the subgroup $\left\{e_{t}\right\}_{t \in \mathrm{R}}$, $\sigma_{2}$ acts as an isomorphism. Since $\sigma_{2}$ is a homeomorphism and since $\left\{e_{t}\right\}_{t \in \mathbb{R}}$ is dense in $X$, it follows that $\sigma_{2}$ is an automorphism of $X$. Moreover, a computation reveals that the adjoint of $\sigma_{2}, \sigma_{2}^{*}$, acting on $\Gamma$ is given by the formula $\sigma_{2}^{*}(\gamma)=\gamma / m$ for all $\gamma$ in $\Gamma$ so that $\sigma_{2}$ is order preserving. Thus $\sigma=\sigma_{1} \sigma_{2}$ is an order preserving affine map of $X$ as was required. For the converse, simply observe that if $\sigma=\sigma_{1} \sigma_{2}$ is an order preserving affine map of $X$ where $\sigma_{2}$ is an automorphism of $X$, then $\sigma_{2}^{*}$ on $\Gamma$ is given by the formula $\sigma_{2}^{*}(\gamma)=\gamma / m$ for all $\gamma$ in $\Gamma$ and some positive number $m$. It follows that $\sigma^{-1} T_{t} \sigma=T_{m t}$; and the proof is complete.

6. Concluding remark. In Theorem I of [8] we showed that if $m$ is an invariant ergodic probability measure on $X$, then $m$ is a representing measure for $\mathfrak{A}$ and $\mathfrak{A}$ is a weak-* Dirichlet algebra in $L^{\infty}(m)$. Independently, Weiss [12] proved a similar theorem for measurable actions of $\mathbf{R}$. It is therefore natural to conjecture that the following analogue of Theorem III is true.

Conjecture. If $m$ is an invariant ergodic probability measure on $X$ and if $U$ is an automorphism of $H^{\infty}(m)$, then there is a bimeasurable, measure preserving, and invertible map $\sigma$ from $X$ onto $X$ and a positive constant $\beta$ such that $\sigma^{-1} T_{t} \sigma=T_{\beta t}$ for all $t$ in $\mathrm{R}$ such that $U \phi=\phi \circ \sigma$ for all $\phi$ in $H^{\infty}(m)$.

It is not difficult to show that every automorphism of $H^{\infty}(m)$ is induced via composition with a bimeasurable, measure preserving, and invertible map $\sigma$; the problem is to show that it intertwines the action in the indicated fashion.

\section{REFERENCES}

1. R. F. Arens, A Banach algebra generalization of conformal mappings of the disc, Trans. Amer. Math. Soc. 81 (1956), 501-513. MR 17, 1226.

2. K. de Leeuw, W. Rudin and J. Wermer, The isometries of some function spaces, Proc. Amer. Math. Soc. 11 (1960), 694-698. MR 22 \#12380. \#2463.

3. R. Ellis, Lectures on topological dynamics, Benjamin, New York, 1969, MR 42

4. F. Forelli, Analytic and quasi-invariant measures, Acta Math. 118 (1967), 33-59. MR 35 \#667.

5. - Conjugate functions and flows, Quart. J. Math. Oxford Ser. (2) 20 (1969), 215-233. MR 40 \#4690. III., 1970.

6. G. Leibowitz, Lectures on complex function algebras, Scott, Foresman, Glenview,

7. S. Merrill III, Maximality of certain algebras $H^{\infty}(d m)$, Math. Z. 106 (1968), 261-266. MR 38 \#2606.

8. P. Muhly, Function algebras and flows. I, Acta Sci. Math. (Szeged) 35 (1973), 111121. 
9. P. Muhly, Function algebras and flows. II, Ark. Mat. 11 (1973), 203-213.

10. - Function algebras and flows. III, Math. Z. 136 (1974), 253-260.

11. M. Nagasawa, Isomorphisms between commutative Banach algebras with an application to rings of analytic functions, Kōdai Math. Sem. Rep. 11 (1959), 182-188. MR 22 \#12379.

12. G. Weiss, Weak*-Dirichlet algebras induced by the ergodic Hilbert transform, L'Analyse Harmonique dans le Domaine Complexe, E. J. Akutowicz, ed., Lecture Notes in Math., vol. 336, Springer-Verlag, Berlin and New York, 1973.

DEPARTMENT OF MATHEMATICS, UNIVERSITY OF IOWA, IOWA CITY, IOWA 52242 